\title{
Electromagnetic MEMS Scanning Mirrors For Holographic Data Storage
}

\author{
Raanan A. Miller, Geoffrey W. Burr, Yu-Chong Tai, and Demetri Psaltis \\ Electrical Engineering, MS 136-93, California Institute of Technology, \\ Pasadena, CA 91125
}

\author{
Chih-Ming Ho \\ MANE, UCLA \\ Los Angeles, CA 90024
}

\author{
Romney. R. Katti \\ Jet Propulsion laboratory \\ Pasadena, CA 91109
}

\begin{abstract}
A mm-sized analog scanning MEMS mirror capable of deflection angles exceeding 60 degrees has been designed and fabricated. Development of this mirror is driven by the need for simple, compact and inexpensive 3-D volume holographic storage systems. Deflection is achieved through the control of an external magnetic field which interacts with a permalloy layer and the current in a 30-turn copper coil. Modeling of the device is also provided here and it agrees with experimental measurements. The use of the mirror is demonstrated in a holographic data storage system and hundreds of holograms have been successfully stored and retrieved.
\end{abstract}

\section{INTRODUCTION}

Volume holographic data storage is achieved by using two laser beams (one "reference" and one "signal" beam) which interfere with each other and form a 3-D grating in a light-sensitive crystal (such as $\mathrm{Fe}$-doped $\mathrm{LiNbO}_{3}$ ). In this process, the interfering beams change the optical properties of the crystal and the crystal stores this 3-D interference grating. To read the data, only the reference beam is needed to shine through the crystal and the prestored 3-D grating inside the crystal reproduces the signal beam. Many multiplexing methods (such as angular, spatial, and wavelength multiplexing) can be used to store a high density of 3$\mathrm{D}$ gratings i.e., holograms, in a limited crystal volume. However, angular multiplexing is the most common and successful technique, of which storage of up to 10,000 holograms in a $1 \mathrm{~cm}^{3}$ crystal has been demonstrated [1]. Holographic data storage is attractive because it has the combined potential of large data storage capacity (hundreds of billions of bytes in a volume roughly that of a compact disk), high bit rate (a billion bits per second) and small random access time $(<100 \mu \mathrm{s})[2]$.

Nevertheless, current volume holographic storage systems are large in size and need a bulky and typically expensive reference beam scanner. Future applications definitely require smaller systems. While most components of current systems such as lenses and lasers can be readily reduced in size, until now there is no commercial beam-steering devices that has the combined merits of low cost, small size and large angle of deflection. Here we then report our work on a MEMS laser-scanning mirror including its behavior as an electromagnetic actuator, and the use of it in a holographic data storage system.

\section{MIRROR DESIGN AND FABRICATION}

Our MEMS scanning mirror (Fig. 1) consists of a plate $\left(4 \times 4 \times 0.04 \mathrm{~mm}^{3}\right.$ in size to accommodate the size of the reference beam) tethered to the bulk silicon substrate by two serpentine springs. Its bottom mirror-like surface (single crystal silicon) serves as the reflector. On the top side of the plate are the electromagnetic components which consist of a 30-turn copper coil and a layer of thin-film permalloy $\left(\mathrm{Ni}_{93} \mathrm{Fe}_{7}\right)$. These two electromagnetic components are used to provide actuation forces.

The fabrication steps (Fig. 2) start with the formation of a $40 \mu \mathrm{m}$ thick epitaxial silicon membrane by anisotropicaly etching the bulk silicon down to a boron etch-stop layer. The boron layer is then removed and an oxide layer is grown for insulation. A $\mathrm{Ti} / \mathrm{Cu}$ seedlayer is next evaporated on the top side of the plate. A photoresist mold is formed and a 30-tum planar copper coil is electroplated ( $9 \mu \mathrm{m}$ thick). Next an insulating photoresist layer is coated, patterned and hard-baked. A Ti/Cu seedlayer is again evaporated, a photoresist mold is formed and an $11 \mu \mathrm{m}$ thick permalloy layer electroplated. Here, a small piece of permalloy (as shown in Fig. 1) serves as a current return path when current is provided to the coil. Finally a thick layer of photoresist is coated and the spring and plate structures are photo-lithographically defined. The exposed oxide layer is removed in BHF and the silicon underneath is Reactive Ion Etched, to free the mirror.
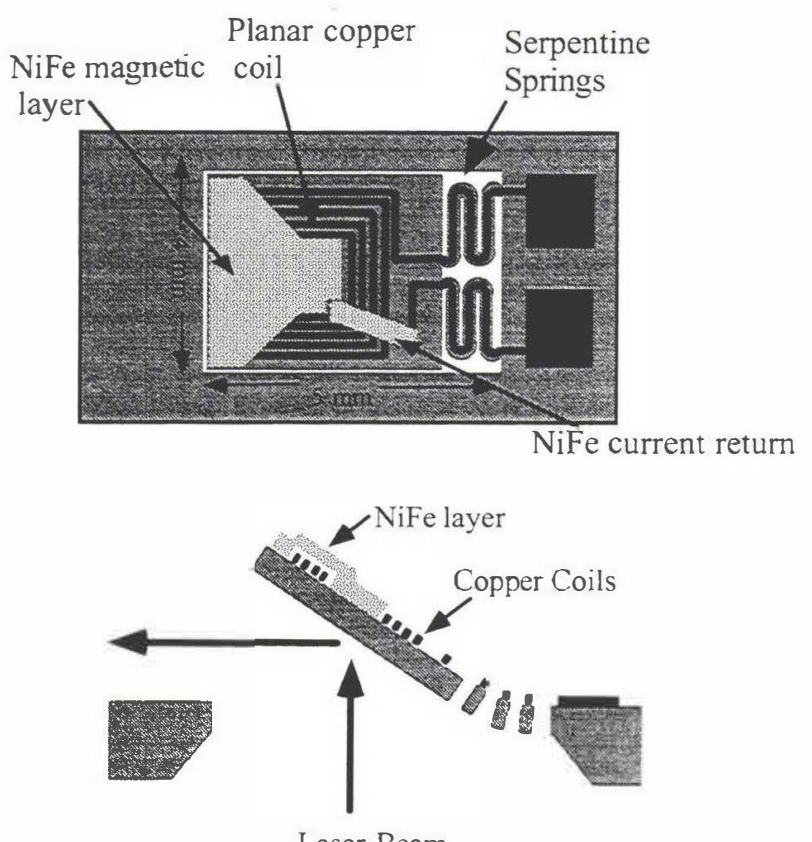

Figure 1. Schematic top view and cross-sectional view (deflected) of a MEMS mirror. The bottom of the plate is used as the mirror surface. 


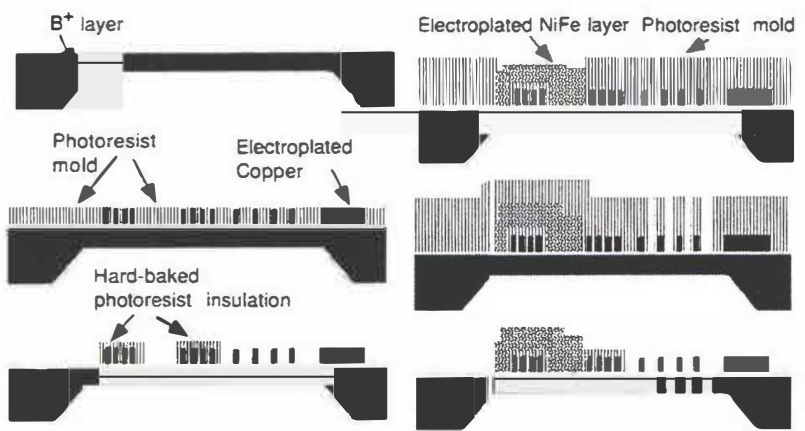

Figure 2. Schematic mirror fabrication steps.

\section{MAGNETIC ACTUATION: THEORY AND EXPERIMENTAL RESULTS}

The MEMS mirror can be operated in two modes. First, with no current supplied to the coils, mirror deflections can be induced by the interaction between an external field and the permalloy layer. The deflections can be controlled by changing the strength and angle of the magnetic field. The second is to fix an external field and use the coil current to deflect the mirror. The external magnetic field will first cause the flap to deflect to a fixed "bias" angle. Deflection about this position can then be induced by changing the magnitude and direction of the current supplied to the coils. If both modes are active, the external field can be conveniently used for the coarse deflection control while the fine positional control is done by the current in the coils. These two actuation control methods are discussed in the following.
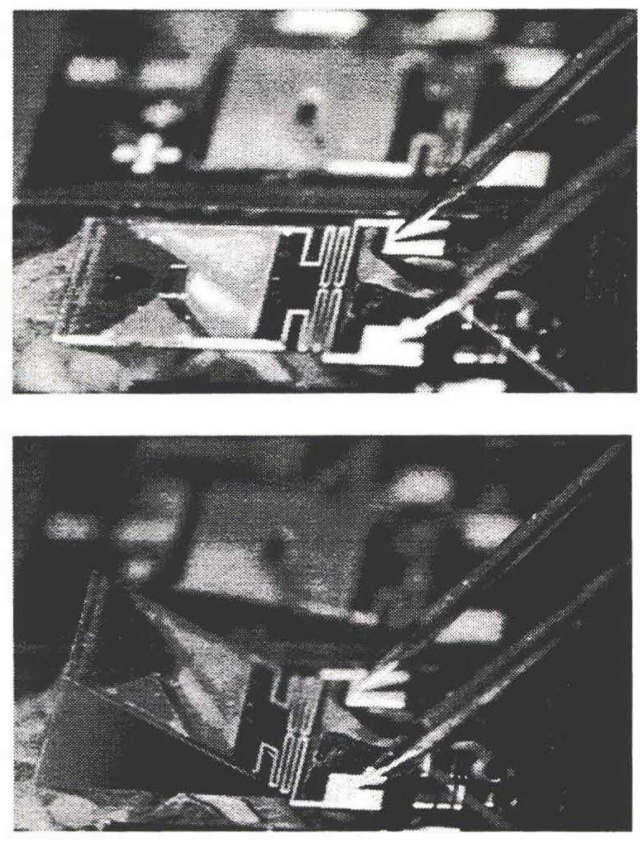

Figure 3. (a) Picture of MEMS mirror in the absence of applied magnetic field and coil current. (b) Actuated mirror in an

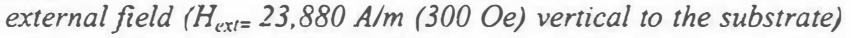
with a coil current of around $30 \mathrm{~mA}$. The deflection is caused by both the permalloy and coil current.

\section{PERMALLOY ACTUATION}

The mirror actuation due to the permalloy/field interaction was investigated by placing the mirror in a uniform magnetic field $H_{c x t}$ (eliminating forces due to field gradients). No current was supplied to the coils. The mirror deflection was then measured for different externally applied field strengths and is shown in Fig. 4. It clearly demonstrates the large deflection $\left(>60^{\circ}\right)$ capability of the mirror.

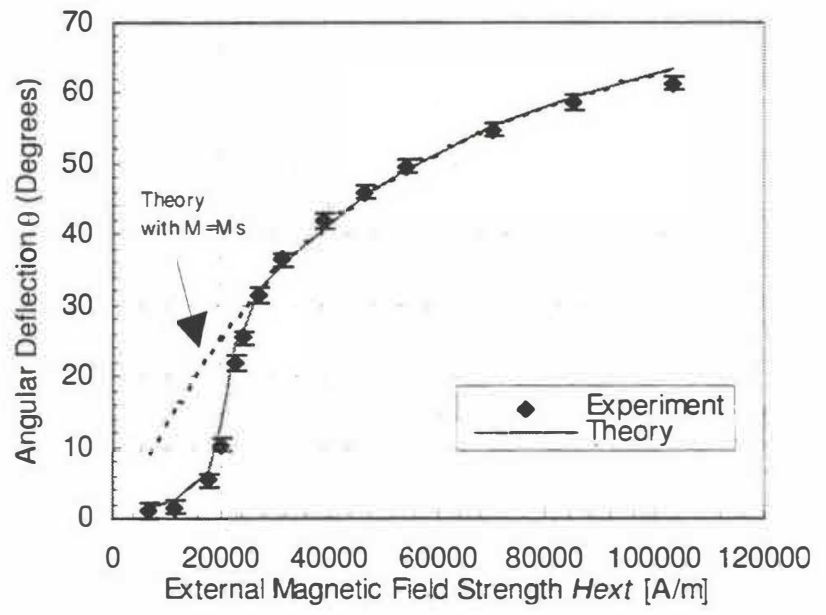

Figure 4. Deflection angle of a MEMS mirror vs. External magnetic field.

The deflection of mirror is modeled using the torque balance (Fig. 5) between a permalloy torque $\left(T_{N i F e}\right)$ and, the restoring torque of the spring $\left(T_{\text {spring }}\right)$. The permalloy torque $T_{N i F e}$ (also described in literature $[3,4])$ is produced by the interaction between $\boldsymbol{B}_{e x t}$ and a magnetic moment $\boldsymbol{m}_{N i F e}$ in the permalloy

$$
T_{N i F e}=\left|\boldsymbol{m}_{N i F e} \times \boldsymbol{B}_{\text {ext }}\right|=V_{\text {NiFe }} M_{N i F e} H_{e x t} \sin (90-\theta)
$$

Here $V_{N i F_{e}}=7.26 \times 10^{-11} \mathrm{~m}^{3}$ is the volume of the permalloy, $H_{c x l}$ is given in $\mathrm{A} / \mathrm{m}, \theta$ is the deflection angle of the flap relative to the substrate and $M_{\mathrm{NiFe}}$ is the magnetization of the permalloy induced by $H_{e x t} \cos (90-\theta)$, the component of $H_{c x t}$ parallel to it. The torque $(\mathrm{Nm})$ is then counter-balanced by the restoring torque of the spring as

$$
T_{\text {spring }}=K \theta
$$

Where $K=4.2 \times 10^{-8} \mathrm{Nm} / \mathrm{deg}$ is the torsional spring constant, and $\theta$ is the deflection angle of the mirror. Eqs. 1 and 2 are then the governing equations used to model the data in Fig. 4, where the theoretical fit (solid line) was generated by equating Eqs. 1 and 2 and solving for $\theta$ under different $H_{e x t}$ values. Here $M_{N i F e}\left(H_{e x x}\right)$ was determined experimentally by BH-loop (Fig. 5) and Vibrating Sample Magnetometer (VSM) measurements performed on the permalloy layer of this particular mirror. The B-H loop gives the shape of $M_{\text {NiFe }}\left(H_{\text {ex }}\right)$, while the VSM measurement determined the saturated magnetization $\left(M_{s}\right)$ of the permalloy to be 0.78 Tesla. An additional curve (dashed line) in Fig. 4 was also plotted with $M_{\text {NiFe }}=M_{s}$. From the point at which this curve and the experimental data diverge, the field $H_{\text {sat }}$ required to saturate the permalloy can be determined. $H_{s a r}$ is simply the component of $H_{e x t}$ in the plane of the permalloy film at this point. It is given by 
$H_{\text {sat }}=27064 \sin 30^{\circ}=13,532 \mathrm{~A} / \mathrm{m}(170 \mathrm{Oe})$. This value is consistent with the value directly read from the BH-loop (Fig. 5).

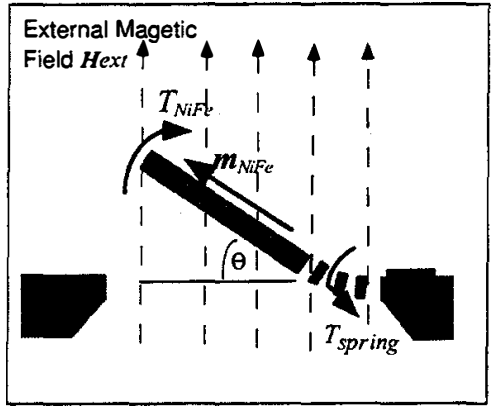

Figure 4. Torque balance of the mirror with permalloy only in an external magnetic field.

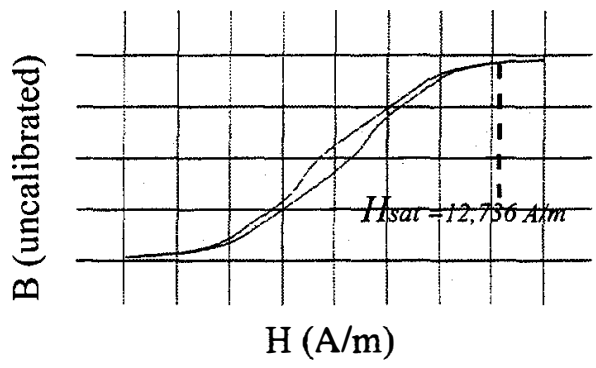

Figure 5. BH-loop measurement of NiFe layer using an shb-109 BH-looper. Horizontal axis has 3,980 A/m per div. (50 Oe /div.).

\section{COIL ACTUATION}

Here, the effect of varying the coil current in a fixed, uniform externally applied field $H_{\text {ext }}$ is discussed. Fig. 6 shows the measured change in deflection angle of the MEMS mirror with an external bias field set to $79,122 \mathrm{~A} / \mathrm{m}$ (994 Oe), which caused the mirror to deflect to $\theta=58^{\circ}$ due to the permalloy $/ H_{c x t}$ interaction described previously. This high field value was used to generate large current-induced deflections and to simplify the analysis by ensuring that the permalloy was saturated.

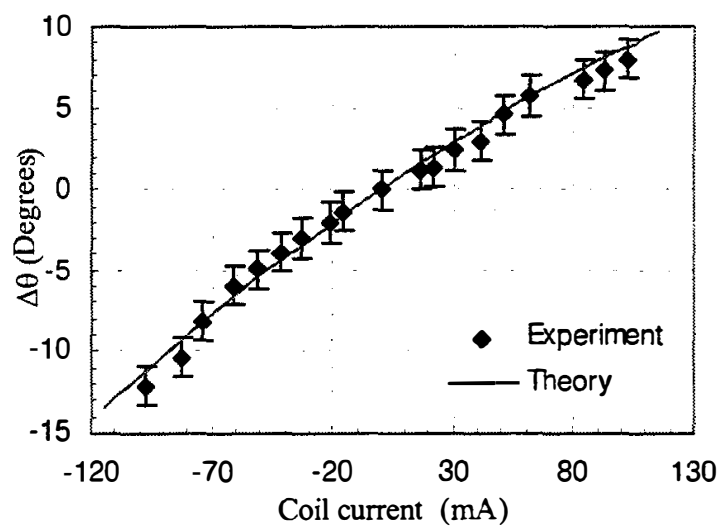

Figure 8. Change in deflection angle from "bias" position at variable coil current with external field of 79,122 A/m (994 Oe).

To model Fig. 8, we have to consider two more terms $T_{\text {coil }}$ and $T_{\text {thermal }}$ in the torque balance equation. First the current in the coils induces an effective magnetic moment $\boldsymbol{m}_{\text {coil }}$ (where $\boldsymbol{m}_{\text {coil }}=I$
$\left.A_{\text {effective }}\right)$. This moment attempts to align with the external field and hence produces a torque on the mirror by[5]

$$
T_{\text {coil }}=\left|\boldsymbol{m}_{\text {coil }} x \boldsymbol{B}_{\text {ext }}\right|=m_{\text {coil }} B_{\text {exl }} \sin \theta
$$

Here $I$ is the current supplied to the coils in amps, $A_{\text {effective }}=0.000132 \mathrm{~m}^{2}$ is the sum of 30 -turn areas, and $B_{\text {ext }}=\mu_{o} H_{\text {ext }}$ is the magnetic flux density in Tesla. Next, the thermal torque is induced by heat generated by this current flowing in the coils. This is modeled by

$$
T_{\text {thermal }}=-C I^{2}
$$

In this case $C=25.2 \times 10^{-6}$ is a fitting constant while $I$ is the applied coil current in amps. The negative sign in Eq. 4 means that the thermal torque always bends the mirror down. Now, when a current is applied to the coils with a constant external magnetic field, the torque balance then becomes

$$
V_{\text {NiFe }} M_{s} H_{e x t} \cos \theta+m_{\text {coil }} B_{e x t} \sin \theta-C I^{2}=K \theta
$$

Eq. 5 has only two unknowns ( $\theta$ and I), which can be solved and used to fit the data in Fig. 8. The result is shown in the solid line and a good fit is again obtained. It is worth noting that the current control can produce a $\pm 10^{\circ}$ deflection which is more than adequate for the scanning mirror application.

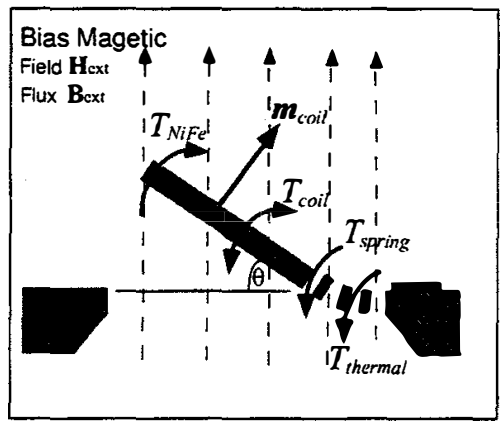

Figure 7. Torque balance of the mirror with both the permalloy and coil current in an external magnetic field.

\section{HOLOGRAPHIC APPLICATION}

In the holographic data storage application the mirror is biased to approximately $45^{\circ}$ in a constant but non-uniform external field ( 200 Oe at mirror), provided by a permanent magnet located $2 \mathrm{~mm}$ above the surface of the mirror. Deflections about this angle (fine control), are induced by varying the coil current.

Two multiplexing schemes are explored. The mirror is first used in an angular multiplexing holographic storage system (Fig. 9a). In this system a single laser beam (Argon laser) is split into a reference beam and a signal beam path. The latter contains an information presentation device (Liquid Crystal Display), a storage crystal, and a detector (CCD camera). The reference beam, is deflected by the MEMS mirror and then passes through a lens system. The lenses keep the beam focused on the same location on the crystal but with different incident angles corresponding the mirrors deflection angle. Fig. 10 shows some typical holograms stored and then retrieved using the MEMS scanning mirror. In addition to the angular multiplexing scheme, the mirror is also used in a combined spatial/angular multiplexing configuration (Fig. 9b). Here, not only is the incident angle of the reference 
beam changed but so is its position on the crystal. The primary advantage of this configuration is that two lenses can be eliminated and the mirror placed right at the crystal surface. Once again for demonstration purposes we stored 100 holograms, all were of good quality.

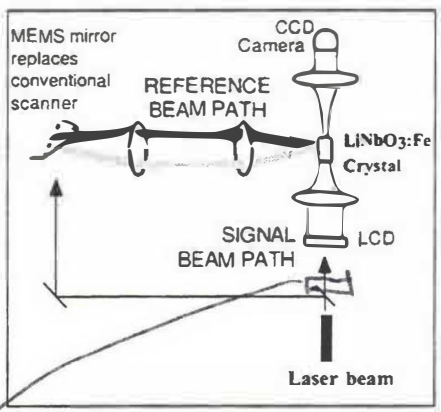

(a)

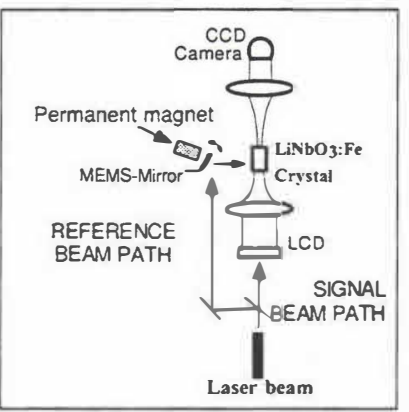

(b) (a) Conventional holographic storage system using commercial beam deflectors. (b) Compact storage system with MEMS mirror.
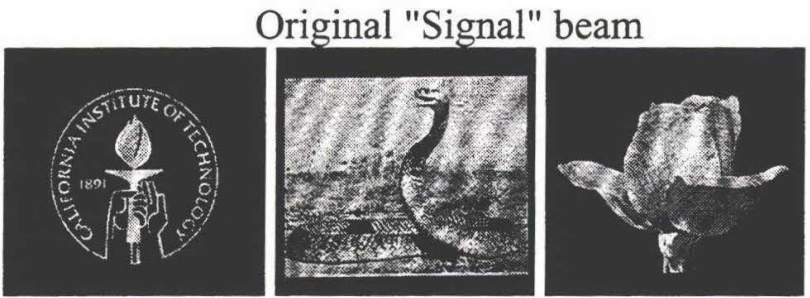

Reproduced Signals (Holograms)
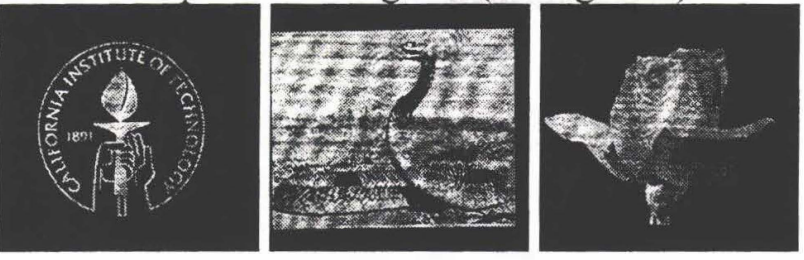

Figure 10. Holograms stored and retrieved, showing original image prior to storage and retrieved image from crystal.

\section{DISCUSSION}

Table 1 shows a comparison of the MEMS mirror with some commercial scanning devices. Although we have been able to successfully demonstrate the storage of hundreds of holograms several aspects of the mirror still need improvement.

First, the $0.03^{\circ}$ spacing between two holograms is only preliminary data. Experimental improvement needs to be done to further reduce this number. Then, the deflection range of $15^{\circ} \mathrm{can}$ be reduced while incorporating the course control. Such an approach can actually reduce necessary current and decrease the undesirable thermal effect. Next, the time response of the mirror is relatively slow. Experimentally, it is found that the mirror has a first mode resonant frequency of about $65 \mathrm{~Hz}$ and a small-angle step response time of $25 \mathrm{~ms}$. It is highly desirable to further reduce this number and future work should increase its damping. Finally, it is apparent that using the MEMS mirror does reduce the overall volume of the demonstrated holographic storage system by more than an order of magnitude. To improve the capacity of the storage then, it is possible to use the saved space to design a multi-mirror array storage system.

\begin{tabular}{|c|c|c|c|}
\hline Device & MEMS mirror & $\begin{array}{c}\text { Commercial } \\
\text { Motorized } \\
\text { stage }\end{array}$ & $\begin{array}{c}\text { Acousto-optic } \\
\text { Deflector }\end{array}$ \\
\hline $\begin{array}{l}\text { Minimum } \\
\text { hologram } \\
\text { spacing }\end{array}$ & $0.03^{\circ}$ & $0.002^{\circ}$ & $0.0015^{\circ}$ \\
\hline $\begin{array}{l}\text { Deflection } \\
\text { range }\end{array}$ & $15^{\circ *}$ & $35^{\circ}$ & $1.5^{\circ}$ \\
\hline $\begin{array}{l}\text { Access time } \\
\text { for } 0.1^{\circ} \\
\text { deflection }\end{array}$ & $25 \mathrm{msec}$ & $130 \mathrm{msec}$ & $70 \mu \mathrm{sec}$ \\
\hline $\begin{array}{l}\text { Reference } \\
\text { beam path } \\
\text { volume** }\end{array}$ & $1-10 \mathrm{~cm}^{3} * * *$ & $686 \mathrm{~cm}^{3}$ & $1372 \mathrm{~cm}^{3}$ \\
\hline $\begin{array}{l}\text { Device } \\
\text { volume }\end{array}$ & $1 \mathrm{~cm}^{3}$ & $60 \mathrm{~cm}^{3}$ & $30 \mathrm{~cm}^{3}$ \\
\hline Cost & Inexpensive & Expensive & Expensive \\
\hline
\end{tabular}

* With current control only.

** Not including laser.

*** For compact configuration as in Fig. $9 b$,

Table 1. Comparison of MEMS mirror with several commercial scanning devices.

\section{CONCLUSION}

A large angle of deflection scanning MEMS mirror has been successfully developed. Its operation with either an external field or a coil current has been studied and modeled. The feasibility of using such a prototype scanning mirror for holographic data storage has been demonstrated.

\section{ACKNOWLEDGMENTS}

This project was partially supported by AFOSR and DARPA under AFOSR Grant No. F49620-94-1-0008. The authors would like to thank Sang Lee and Gordon Hughes of Seagate technology, and Stanley Bacon for their help with magnetic measurements.

\section{REFERNCES}

1. G. W Burr , F. H. Mok and D. Psaltis "Large scale volume holographic storage in the Long Interaction Length Architecture", SPIE proceedings, paper 2297-47, (1994).

2. D. Psaltis and F. H. Mok, "Holographic Memories", Scientific American, pp. 70-75, November (1995).

3. J. W. Judy, R. S. Muller, "Magnetic microactaution of torsional polysilicon structures", Transducers 95, Stockholm, Sweden, 6/256/29, pp. 332 - 335, (1995).

4. C. Liu, T. Tsao, Y.C. Tai, J. Leu, et. al, "Out-of plane permalloy magnetic actuators for delta-wing control", Proceedings MEMS 95, Amsterdam, The Netherlands, 1/29-2/2, pp. 328-331, (1995).

5. J. D. Kraus, "ELECTROMAGNETICS", fourth Edition, McGraw-Hill, 1988, pp. 304 - 307. 